\title{
APOGEE: A high resolution SDSS-III H-band survey of the Milky Way
}

\author{
Matthew Shetrone ${ }^{1}$ \\ University of Texas, McDonald Observatory \\ E-mail: shetronelastro.as.utexas.edu
}

James Lawler

University of Wisconsin

Ricardo Schiavon

Gemini Observatories

Steve Majewski
Fred Hearty
John Wilson
University of Virginia

\section{Carlos Allende Prieto}

Instituto de Astrofisica de Canarias

Jeniffer Johnson

Ohio State University

\section{Jon Holtzman}

New Mexico State University

\section{Peter Frinchaboy}

Texas Christian University

The Sloan Digital Sky Survey III/Apache Point Observatory Galactic Evolution Experiment (SDSS-III/APOGEE) is a large-scale spectroscopic survey of Galactic stars. SDSS-III/APOGEE will obtain high-S/N, R 30,000 spectra, covering the 1.51 to 1.68 micron range for of order 100,000 stars. APOGEE will target stars in all components of the Galaxy, including the halo, bulge, disk, and tidal streams. APOGEE will be the deepest chemodynamical survey of the bulge and disk stellar populations, and amass substantial samples from the other Galactic components. With abundances for at least 15 elements accurate to $\sim 0.1$ dex and radial velocity accuracies of $\sim 0.3 \mathrm{~km} / \mathrm{s}$, APOGEE will provide fundamental constraints on galaxy evolution models and lend insights into the physical processes leading to the formation of the Galaxy.

11th Symposium on Nuclei in the Cosmos - NIC XI

Heidelberg, Germany

July 19-2320

$1 \quad$ Speaker 


\section{APOGEE in a NUTSHELL}

- Dedicated 300 fiber, cryogenic, near-infrared spectrograph.

- $\quad R \sim 27,500-31,000, S / N \sim 100 /$ pixel, H-band (1.51-1.68m) spectra of $\sim 10^{5}$ field and cluster red giants down to $H \sim 12.5$.

- Also, some additional deeper $(H \sim 14.1)$ and special target probes.

- Majority fraction of bright-time nights on the SDSS 2.5-m telescope over 3 years of SDSSIII starting in April 2011.

- Accurate radial velocities (to $0.3 \mathrm{~km} / \mathrm{s}$ ) and precision (0.1 dex) abundance patterns for the entire sample.

- Focus on $H$ band allows deeper probe of the dust-extinguished Galactic bulge and disk than any other survey planned.

- At $H=13.5$, can reach giant stars as far as $70 \mathrm{kpc}$ in Galactic halo and $25 \mathrm{kpc}$ through $A_{H}=2.5\left(A_{V}=15\right)$ of dust.

- Red giant/clump stars reliably selected from $2 M A S S$.

- Will increase by orders of magnitude the number of available high-resolution stellar spectra, especially at low $b$ (Fig. 1).

- Apart from critical elements $\mathbf{C}, \mathbf{N}, \mathbf{O}$ and Fe, APOGEE will derive abundances of $\alpha \mathbf{( M g}$, Si, S, Ca, Ti), odd-Z (Na, Al, K), Fe-peak (V, Mn, Ni, Co, Cr) and possibly neutroncapture (Ce, $\mathbf{R b}$ ?) elements with a pipeline reduction code.

- Public data releases will include fully reduced and calibrated spectra as well as pipelinederived, high level data (RVs, RV variability, stellar parameters, abundances) for all stars.

\section{APOGEE Science Goals}

- First 3-D MW chemical abundance distribution (for $\sim 15$ elements).

- Metallicity distribution functions across disk, bulge, halo.

- Constrain the IMF and SFR of bulge/disk as function of radius, metallicity/age, chemical evolution of inner Galaxy.

- Determine nature of Galactic bar and spiral arms and their influence on abundances/kinematics of disk/bulge stars.

- Measure Galactic rotation curve from stars of known distance.

- Search for/probe chemistry/kinematics of halo substructure, especially at low-latitude -e.g., the Monoceros Ring.

- Combine APOGEE spectroscopic parallaxes with optical, NIR and MIR data to map Galactic dust distribution and constrain variations in the extinction law.

- Systematic probe of Galactic star clusters. 

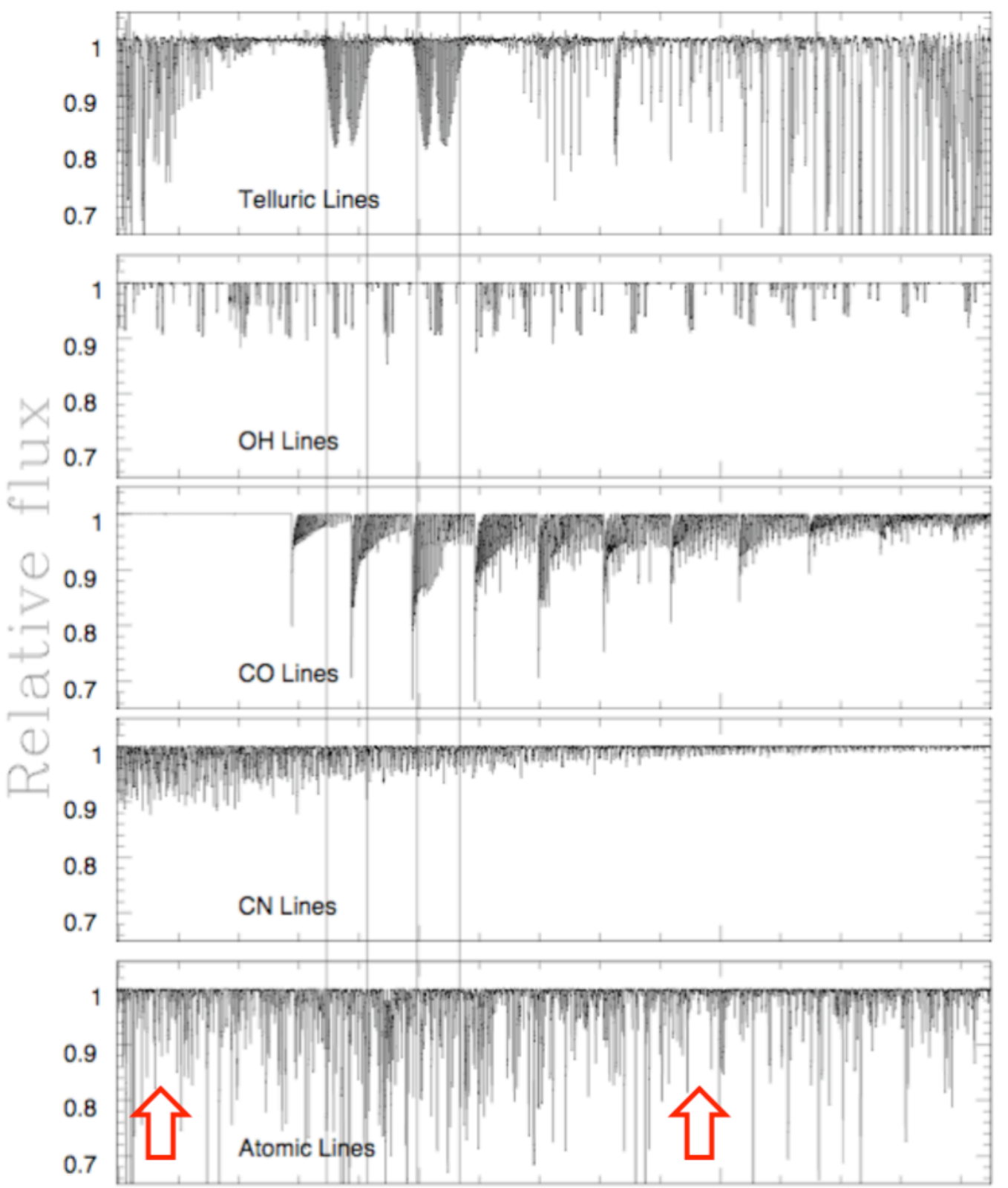

$1.5 \times 10^{4}$

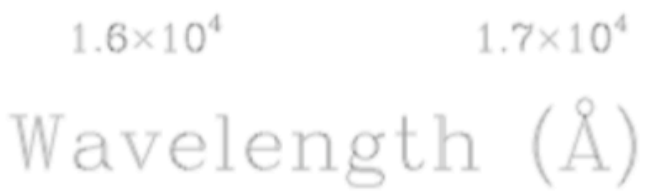

Figure 1 High resolution spectra of the sky transmission (top) and of different molecular and atomic species. The red arrows indicate nominal APOGEE spectral windows, designed to minimize telluric absorption and maximize chemical information. 


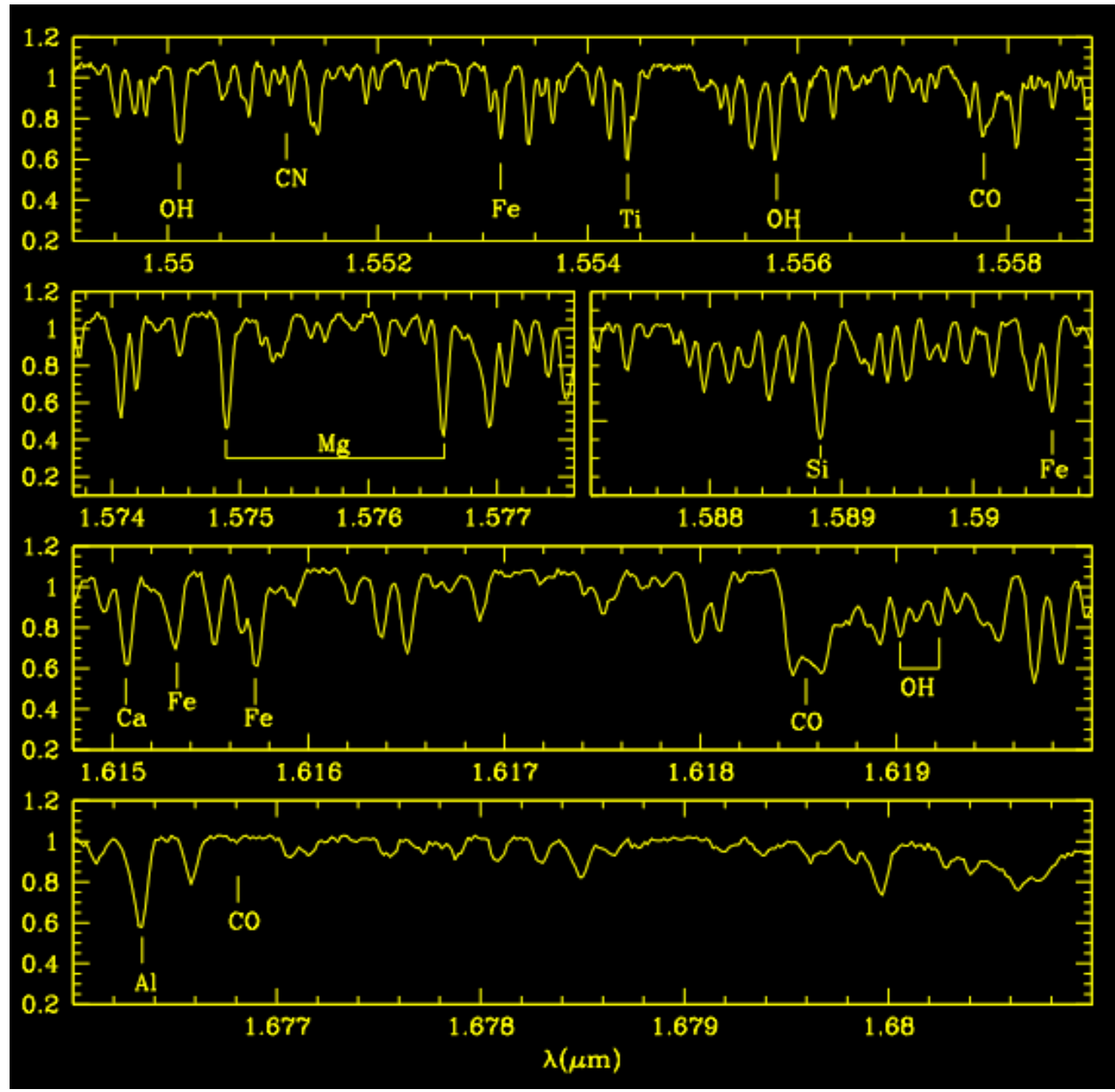

Figure 2 Simulated $S / N \sim 100$, H-band spectrum for typical solar metallicity K-giant star in a few spectral windows of interest (totaling $\sim 15 \%$ of APOGEE coverage), which show well resolved spectral lines for some chemical species to be probed by APOGEE.

\section{Current Status of APOGEE}

- Funded by NSF, Sloan Foundation, and SDSS-III collaboration.

- APOGEE spectrograph in construction stage.

- APOGEE spectrograph building constructed on-site.

- Data reduction and stellar parameters/abundance analysis pipelines under construction.

- Main survey field selection being finalized.

- Target selection criteria being finalized.

- First light on telescope expected Dec 2010.

- First survey observations expected April 2011. 